\title{
IMPROVEMENT OF DNA MICROARRAY BIOCHIPS USING MICROFLUIDIC MIXING TECHNIQUE
}

\author{
Robin H. Liu, Ralf Lenigk, Dale Ganser, Justin Bonanno, and Piotr Grodzinski \\ Microfluidics Lab, PSRL, Motorola Labs \\ Tempe, AZ 85284 \\ Bobbi Sanchez and Pankaj Singhal \\ Steve Dai \\ Motorola Life Science \\ Tempe, AZ 85284 \\ SSRC, Motorola Labs \\ Tempe, AZ 85284
}

\begin{abstract}
Conventional DNA microarray hybridization relies on the diffusion of targets to surface-bound probes, and thus is a ratelimited process. In this paper, a micromixing technique based on cavitation microstreaming principle was developed to accelerate hybridization process. Fluidic experiments showed that the time to fully mix a $20 \mu \mathrm{L}$ chamber using microstreaming was significantly reduced from hours (a pure diffusion-based mixing) to tens of seconds. Cavitation microstreaming was implemented to enhance DNA hybridization in both fluorescent detection based and electrochemical detection based microarray chips. Hybridization results showed that microstreaming results in up to 5 folds signal enhancement and kinetics acceleration, and signal uniformity is also significantly improved, compared to conventional diffusionbased biochips. Acoustic microstreaming has many advantages over most existing mixing techniques for hybridization enhancement, including simple apparatus, ease of implementation, low power consumption $(2 \mathrm{~mW})$, and low cost.
\end{abstract}

\section{INTRODUCTION}

DNA microarray is one of the most promising analytical techniques in molecular biology. DNA hybridization is a heterogeneous bimolecular reaction, involving mixing and binding of the target DNA in a free solution and the DNA probes immobilized on a surface. The hybridization rate depends on a number of parameters, among which mass transfer in the bulk (3D) as well as on the surface (2D) is playing an important role. In most conventional microarray biochips, hybridization solely relies on diffusion, and thus is a lengthy rate-limiting process $(6 \sim 20 \mathrm{hrs})$ [1]. This greatly limits the throughput of sample analyses. Various methods have been developed to accelerate the hybridization process, including electric hybridization [2], dynamic hybridization using paramagnetic beads [3], and the Flow-Thru approach [4]. It is believed that introduction of micromixing technique can accelerate hybridization kinetics and improve uniformity of hybridization.

A few interesting mixing techniques to facilitate mixing of various fluids within a DNA hybridization chamber have been reported in recent years. Of particular interest is "rotational mixing" that involves a rotatable body having a rotational axis [21]. This technique generally works better in macro-scale fluidic chambers since the mixing relies on the inertial force of the fluid. However, when the reaction chamber is shallow (e.g., $\sim 200 \mu \mathrm{m}$ or less), viscous force dominates fluidic behavior due to low Reynolds number $(\sim 1)$. It is believed that mixing in microfluidic environment cannot be significantly enhanced by mechanical agitation means, such as rotation or shaking.
Ultrasonic mixing using a piezoelectric element was also reported to achieve hybridization enhancement [5]. Other similar works on ultrasonic mixing were reported in [6] and [7]. The former used ultrasonic traveling waves generated by a pieozoelectric film to the liquid in a mixing chamber. The latter used loosely-focused acoustic waves generated by an electrodepatterned pieozoelectric film. Both devices require a complicated fabrication process (e.g., Si bulk etching). Other mixing approaches to enhance hybridization include bubbling gas through the chamber and "drain and fill" method [8]. Both mixing schemes require system setups with in-line flowing and pumping, and possibly a precise flow control.

In this paper, we will describe the phenomenon of cavitation microstreaming, which provides a mechanism for achieving rapid and homogeneous mixing in a hybridization chamber. An air bubble trapping design using micromachined air pockets, coupled with a commercially available piezoelectric (PZT) disk, is presented. Fluidic experiments to demonstrate utilization of cavitation microstreaming to achieve a high degree of fluid transport and mixing enhancement in a hybridization chamber will be described. DNA hybridization experiments to demonstrate improvement of hybridization kinetics and signal uniformity using cavitation microstreaming will be discussed.

\section{THEORY}

An air bubble in a liquid medium can act as an actuator when the bubble undergoes vibration within a sound field. The behavior of bubbles in sound fields is determined largely by their resonance characteristics. For frequencies in the range considered here $(\sim$ $\mathrm{kHz})$, the radius of a bubble at resonant frequency $\mathrm{f}(\mathrm{Hz})$ is given by the equation:

$$
2 \pi a f=\sqrt{3 \gamma P_{o} / \rho}
$$

where $a$ is the bubble radius (cm), $\gamma$ is the ratio of specific heats for the gas, $P o$ is the hydrostatic pressure (dynes/cm2) and $\rho$ is the density of the liquid $(\mathrm{g} / \mathrm{cc})$. Using this equation with the parameter values $f=5000 \mathrm{~Hz}$, one finds $a$ for resonance to be approximately $0.3 \mathrm{~mm}$.

When the bubble undergoes vibration within a sound field, the frictional forces generated at the air/liquid interface induce a bulk fluid flow around the air bubble, called cavitation microstreaming or acoustic microstreaming [9]. It was found that cavitation microstreaming is orderly at low driving amplitudes when the insonation frequency drives the bubbles at their resonance frequency for pulsation and when the bubbles are situated on solid boundaries. The bubble-induced streaming is strongly dependent on 
frequency for a given bubble radius, and on bubble radius for a given frequency. Acoustic microstreaming arising around a single bubble excited close to resonance produces strong liquid circulation flow in the liquid chamber. This liquid circulation flow can be used to effectively enhance mixing. Although cavitation microstreaming has been reported and studied since 1950s [9], we have not found the use of this phenomenon to enhance micromixing. One challenge here is to precisely control the size of the air bubbles. In this work, we have developed a design to engineer the air bubbles for mixing enhancement.

\section{EXPERIMENTAL DETAILS}

Fluidic experiments with dye were implemented to visualize and study cavitation microstreaming in a hybridization chamber. As shown in Fig.1, the chamber was constructed by sealing a conventional DNA microarray glass chip with a polycarbonate cover layer using a double-side adhesive tape (3M, St. Paul, MN). The adhesive tape with thickness of $100 \mu \mathrm{m}$ serves as a spacing gasket to define the shape and dimension of the chamber. The cover layer has a desired number of air pockets uniformly distributed above the chamber $(12 \times 15 \times 0.1 \mathrm{~mm})$. The air pockets (500 $\mu \mathrm{m}$ in depth and $500 \mu \mathrm{m}$ in diameter) that were milling machined were used to trap air bubbles in the reaction solution. A PZT disk (15 mm diameter, APC Inc., Mackeyville, PA) was glued on the outer surface of the cover layer. The chamber contents were irradiated by the sound that came from the PZT. Visual observations were made from above using a stereoscope. One half of the chamber was filled with DI water and the other half with a red dye solution that was used to depict motion of fluid elements in the chamber. The frequencies employed were $3 \mathrm{kHz}$ (sinusoidal wave) with $10 \mathrm{Vpp}$.

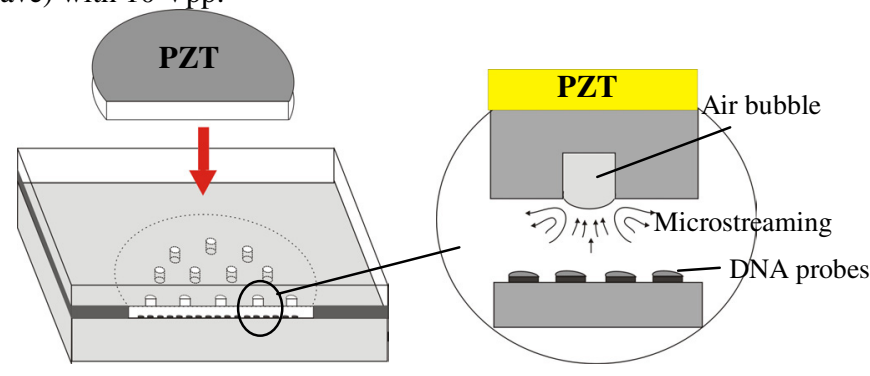

Fig.1 Schematic of the mixing chamber with DNA probes.

High-density DNA microarray hybridization was performed to evaluate mixing enhancement in improving hybridization efficiency and uniformity over conventional diffusion-based hybridization. A fluorescent detection based microarray biochip that consists of a high-density array of oligonucleotide probes dispensed on a 1x3 inch pre-treated glass slide was used. The oligonucleotide probes that consist of two types of oligonucleotides (NEO and YJEK) and a positive control were arranged in a uniform pattern across the entire slide. A 1 x 3 inch double-side adhesive tape (3M, St. Paul, MN), which was cut into four 12 x 15 mm windows, was used to bond a polypropylene cover layer with the glass slide and serves as a spacing gasket to define the shape and dimension of the chambers on the glass slide. The polypropylene cover layer consists of a number of air pockets (500 $\mu \mathrm{m}$ in depth and $500 \mu \mathrm{m}$ in diameter) on the side facing the DNA array. The air pockets are uniformly distributed across the cover layer with a pitch of $2 \mathrm{~mm}$. A PZT disk (15 mm diameter) was glued on the outer surface of one chamber, in which cavitation microstreaming was implemented while static hybridization (i.e., diffusion-only) was performed as a control in one of the other three chambers on the same chip. During hybridization, a fluorescently labeled oligonucleotide target solution (with a volume of $18 \mu \mathrm{L}$ ), which has 50\% formamide, 6xSSPE, and $10 \mathrm{nM} \mathrm{Cy3}$ labeled targets that have complements to the NEO and YJEK sequence on the slide, was loaded into each detection chamber. The PZT was driven at $5 \mathrm{kHz}$ and $10 \mathrm{Vpp}$. Hybridization was carried out for $2 \mathrm{hr}$. Following the hybridization, the polypropylene layer was removed from the array glass slide, which was subsequently washed with TNT for 30 minutes at $42{ }^{\circ} \mathrm{C}$ and then $3 \mathrm{x}$ water. The glass slide was scanned using a microarray scanner (Axon Instruments, Inc., Union City, CA).

An assay for single nucleotide polymorphisms (SNP's) associated with hematochromatosis was performed in a Motorola eSensor $^{\mathrm{TM}}$ device with microstreaming. The eSensor ${ }^{\mathrm{TM}}$ devices allow for continuous measurement of DNA hybridization during the reaction due to the homogenous nature of the assay, thus allowing hybridization kinetics study. Each device consists of a plastic cover layer assembled with a printed circuit board (PCB) chip that has 16 detection electrodes. Four electrodes contain identical oligonucleotide probes for HFE-H. The plastic cover layer has $4 \times 4$ air pockets $(500 \mu \mathrm{m}$ in depth and $500 \mu \mathrm{m}$ in diameter) facing the DNA probes. A PZT disk was glued on the outer surface of the cover layer to induce microstreaming during the hybridization. The DNA target solution containing HFE-H polymorphism (210 bp) was amplified from human genomic DNA characterized for HFE genotype. The PCR product was prepared by asymmetrical amplification of $100 \mathrm{ng}$ of human genomic DNA using a set of three primers with a final concentration of $0.5 \mu \mathrm{M}$ per primer, $400 \mu \mathrm{M}$ dNTP, $50 \mathrm{mM} \mathrm{KC,} 10 \mathrm{mM}$ Tris-HCL (pH 8.3), 2 $\mathrm{mM} \mathrm{MgCl} 2,0.05 \mathrm{U} / \mu \mathrm{l}$ Taq polymerase, and $100 \mu \mathrm{g} / \mathrm{ml}$ bovine serum albumin. Cycling parameters were: $95^{\circ} \mathrm{C}(3 \mathrm{~min})$ to denature human DNA, followed by 40 cycles $\left(94^{\circ} \mathrm{C}\right.$ for $45 \mathrm{sec}, 58^{\circ} \mathrm{C}$ for 55 sec, $72^{\circ} \mathrm{C}$ for $60 \mathrm{sec}$ ), and ending $72{ }^{\circ} \mathrm{C}$ for $6 \mathrm{~min}$ to extend all unfinished DNA strands. Following PCR, the amplicon solution was mixed with a hybridization solution in a ratio of $1: 2$. The hybridization buffer is composed of $0.85 \mathrm{M}$ sodium perchlorate, $18.4 \%$ v/v Qiagen Lysis buffer AL, $10 \mathrm{mM}$ tris $\mathrm{HCl} \mathrm{pH} \mathrm{7.5,} 1 \mathrm{mM}$ 6-mercaptohexnol and $10 \% \mathrm{v} / \mathrm{v}$ fetal bovine serum. The hybridization cocktail was then filled into an eSensorTM chip with an internal volume of $65 \mu \mathrm{L}$. Hybridization was performed at $35^{\circ} \mathrm{C}$. During the hybridization process, the PZT was driven at $5 \mathrm{kHz}$ and 10 Vpp. The signals were read out using a Motorola Hydra®600 instrument. For comparison purpose, hybridization reaction was also implemented in a conventional diffusion-based eSensor ${ }^{\mathrm{TM}}$ chip using the same amplicon mixture. Study of hybridization kinetics as a function of cavitation microstreaming amplitude (Vpp) was also performed using $5 \mathrm{Vpp}$ and $40 \mathrm{Vpp}$, as compared to $10 \mathrm{Vpp}$.

\section{RESULTS AND DISCUSSION}

Fluidic dye experiments showed that sonic irradiation caused little motion of the liquid if air bubbles were excluded from the chamber. However, with air bubbles that have a resonant frequency matching the PZT frequency, a gross liquid motion was seen to take place around individual bubble. Since the top pockets are distributed above the chamber, the resulting acoustic streaming dominates the mixing in the whole chamber within a few seconds. 
As shown in Fig. 2, when the PZT was turned on $(5 \mathrm{kHz}$ and 10 $\mathrm{Vpp}$ ), the streaming occurred around each air bubble and the microstreaming fields began to interfere with each other. Churning motion in the liquid was seen at the air-liquid interface. Fluidic elements were focused into a narrow stream and move rapidly toward the bubble surface, then decreases as the elements spread out and leave the bubble region. As streaming continued and fluid elements moved rapidly, the dye eventually completely filled the chamber. Fluidic experiment (Fig. 2) shows that complete mixing was achieved across the whole chamber within $1 \mathrm{~min}$ and $45 \mathrm{sec}$, while the mixing based on pure diffusion (i.e., without acoustic mixing) took about $12 \mathrm{hrs}$ for the same chamber. Dye experiments were also performed to investigate the relationship between the mixing rate and acoustic parameters. It was found that square sound wave gives faster mixing than sinusoidal sound wave. Higher amplitude also results in faster mixing. For example, complete mixing in the same chamber was achieved within $35 \mathrm{sec}$ if a $5 \mathrm{kHz}$ and $40 \mathrm{Vpp}$ sinusoidal wave was employed.

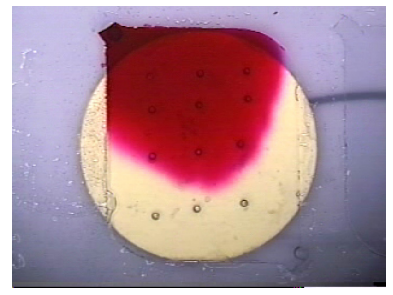

(a)

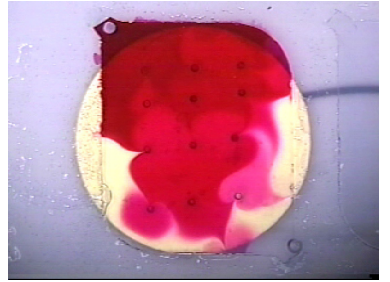

(c)

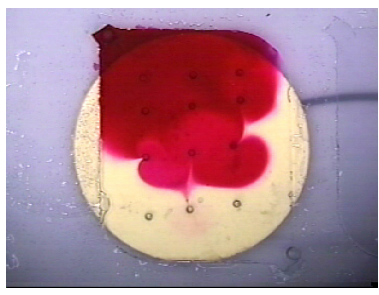

(b)

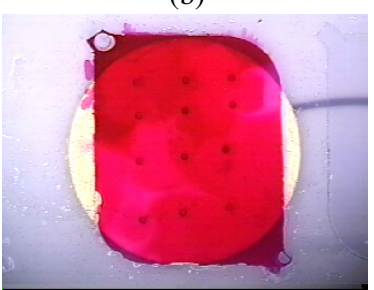

(d)
Fig.2: Images showing cavitation microstreanming in a $12 \times 15 \mathrm{x}$ $0.1 \mathrm{~mm}$ chamber at (a) time 0 ; (b) $28 \mathrm{sec}$; (c) $1 \mathrm{~min} 7 \mathrm{sec}$; (d) $1 \mathrm{~min}$ $46 \mathrm{sec}$.

Fig. 3 shows fluorescent scanning images of a microstreaming enhanced array and a diffusion-based array. Fluorescent intensity data for both arrays were analyzed. As shown in Fig.4, the average intensity of the mixing array is five times more than that of the static hybridization array, and signal uniformity (co-variance) is greatly improved by implementing microstreaming. These results indicate that hybridization reactions in oligonucleotide array formats can generally be affected by the level of mixing of the target ligand. Efficient and effective cavitation microstreaming can ensure maximal presentation of the sample targets to the array, and thus significantly improve hybridization efficiency and quality.
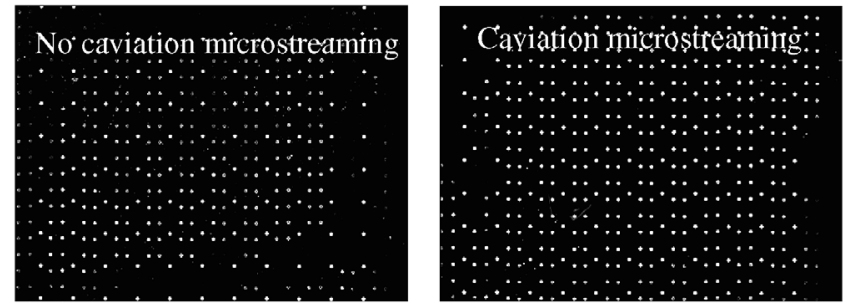

Fig. 3: Fluorescent images of hybridization assay in a HD array chip.

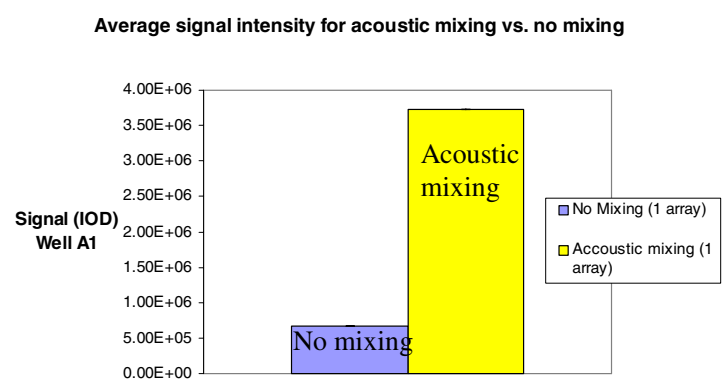

(a)

Uniformity of signal intensity for acoustic mixing vs. no mixing

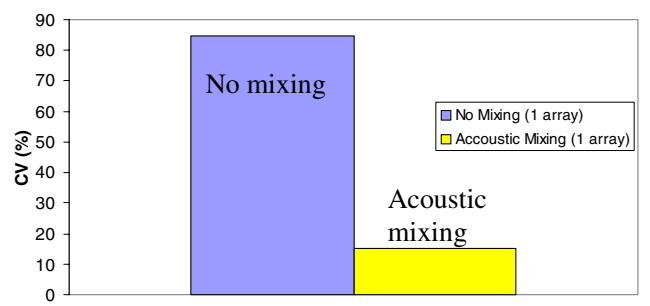

(b)

Fig.4: (a) Averaged fluorescent intensity. (b) Intensity uniformity (note: $0 \% \mathrm{CV}=$ fully uniform).

Kinetic data of the genotyping experiments using target DNA obtained by PCR amplification from human genomic DNA to bind to the sensor electrodes on Motorola eSensor ${ }^{\mathrm{TM}}$ chips were collected by measuring electrochemical signal as a function of time. Fig. 5 summarizes the hybridization kinetics results for a mixing-enhanced device and a diffusion-based device. Each data point is the mean value obtained from four electrodes with identical DNA capture probes in the same device. Note that the $y$-axis in the figure is the measurement of the Faradaic current from the electrodes. The Faradaic current is directly proportional to the number of ferrocene moieties immobilized at the electrode surface that in turn is proportional to the number of target nucleic acid molecules. The results show that for diffusion-based hybridization, hybridization signal evolves slowly and exhibits linear increase. This steady-state behavior indicates the attainment of equilibrium for the hybridization reaction is not achieved within the time frame of the experiment. Moreover, the standard deviation of each data point shows that the pure diffusion-based hybridization has relatively large electrode-to-electrode variation. For the hybridization assay coupled with cavitation microstreaming, the signal increased rapidly, showing additional acceleration and much uniform signal distribution (small standard deviations) compared to the diffusion-based device. After 40 minutes of hybridization, the sample in the mixing-enhanced device reached a saturated Faradaic current value with a signal-to-background ratio of 30 , which is 5 times that of the sample in the diffusion-based device. It took approximately $6 \mathrm{hrs}$ for the diffusion-based sample to reach the saturated level (not shown here). 
HFE-H Amplicon Hybridization Kinetics PZT mixing vs. Diffusion

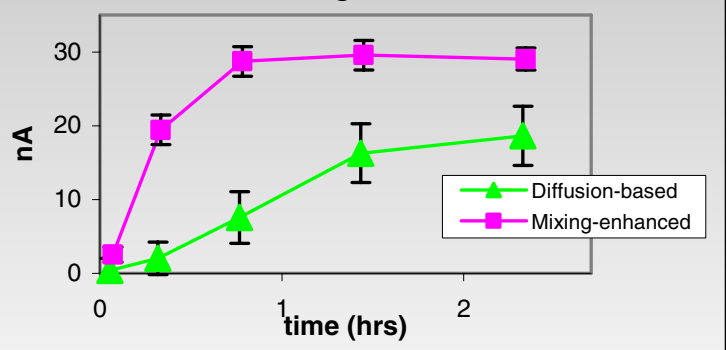

Fig. 5: Hybridization kinetics measurement for acoustic mixing vs. diffusion in eSensor ${ }^{\mathrm{TM}}$.

From fluidic experiments with dye, it has been found that higher acoustic amplitude (i.e., peak-to-peak voltage) gives faster mixing enhancement. To evaluate hybridization enhancement as a function of acoustic amplitude, HFE-H hybridization was also performed in eSensor ${ }^{\mathrm{TM}}$ devices with different amplitudes. As shown in Fig. 6, $40 \mathrm{Vpp}$ results in faster hybridization kinetics and more uniform signals than 10 and $5 \mathrm{Vpp}$.

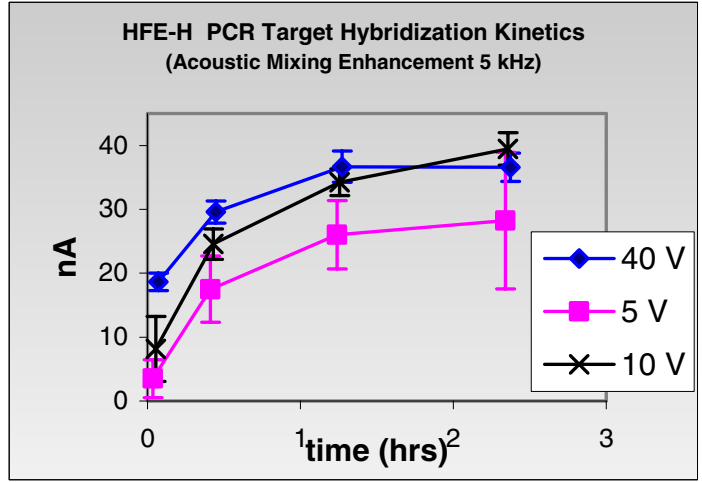

Fig. 6: Hybridization kinetics for chips using different acoustic amplitudes (V peak-to-peak).

Both fluidic and hybridization experiments demonstrated that cavitation microstreaming not only provides rapid lateral mass transport of fluidic elements (thus ensures a homogenous mixture of targets across the ship) but also enhances the vertical mass transport of target DNA in the solution to the probes on the surface. The rapid lateral fluidic movement allows uniform hybridization signals to be achieved across the large surface area on the chip. Uniformity of hybridization is critical especially for detection of low-abundance targets. Lack of lateral flow convection could lead to non-homogeneous array performance and hybridization differences that are independent of differences in target concentration.

The fast vertical mass transport of target DNA in the solution to the surface-bound probes gives rise to hybridization kinetics acceleration. Cavitation microstreaming in a shallow hybridization chamber reduces the thickness of the stagnant (diffusion) boundary layer of fluid on the chip surface, and thus decreases depletion effect. Targets are in close proximity to the surface-bound probes, resulting fast mass transport due to short diffusion length. The combination of rapid lateral and vertical mass transport allows continuous replenish of fresh DNA targets around the probes that has been depleted of complementary targets. As a result, the hybridization rate is greatly increased. Although this hybridization enhancement is not as significant as compared to the flow-thru approach [4] and the electronic DNA hybridization [10], the distinct advantage that cavitation microstreaming has over the above two methods is its rapid lateral mass transport that significantly enhances uniformity of hybridization. Moreover, cavitation microstreaming requires very limited and simple mixing apparatus, and thus can be easily implemented in most existing biochip devices including conventional high-density or low-density DNA/RNA array chips.

\section{CONCLUSIONS}

We have developed a microfluidic mixing technique that is based on the principle of cavitation microstreaming. We demonstrated that rapid and homogeneous dye mixing was achieved in a shallow chamber device. Hybridization experiments in high-density microarray chips and $\mathrm{eSensor}^{\mathrm{TM}}$ devices showed that cavitation microstreaming results in 5-fold increase in hybridization rate and significantly enhanced signal uniformity due to significant convective current in both lateral and vertical directions, compared to conventional diffusion-based hybridization.

\section{ACKNOWLEDGEMENT}

The authors would like to express their thanks to Dr. Gary Olsen from Motorola Life Science for useful discussion on hybridization kinetics. This work has been sponsored in part by DARPA contract \#MDA972-01-3-0001 and NIST ATP contract \#1999011104A.

\section{REFERENCES}

1. Schena, M., Microarray BiochipTechnology. 2000, Natick, MA: Eaton Publishing.

2. Sosnowski, R., et al., Rapid Determination of Single Base Mismatch Mutations in DNA Hybrids by Direct Electric Field Control. Proc. Natl. Acad. Sci., 1997. 94: p. 1119-1123.

3. Fan, Z.H., et al., Dynamic DNA hybridization on a chip using paramagnetic beads. Anal. Chem., 1999. 71: p. 4851-4859.

4. Cheek, B.J., et al., Chemiluminescence Detection for Hybridization Assays on the Flow-Thru Chip, a ThreeDimensional Microchannel Biochip. Anal. Chem., 2001. 73: p. 5777-5783.

5. US patent No. 6,168,948 B1, Anderson, R. C., et al., "Miniaturized Genetic Analysis Systems and Methods", 2001

6. Moroney, R.M., R.M. White, and R.T. Howe. Ultrasonically induced microtransport. in MEMS '95. 1995. The Netherlands.

7. Zhu, X. and E.S. Kim, Microfluidic Motion Generation with Acoustic Waves. Sensors and Actuators: A. Physical, 1998. 66 (1-3): p. 355-360.

8. US Patent No. 6,114,122, Besemer, D., et al., "Fluidics Station With a Mounting System and Method of Using”, 2000.

9. Elder, S.A., Cavitation Microstreaming. J. Acoust. Soc. Am., 1959. 31: p. 54-62.

10.Edman, C., et al., Electric Field Directed Nucleic Acid Hybridization on Microchips. Nucl. Acids Res., 1998. 25: p. 4907-4914. 